\title{
Transplante de Pâncreas e llhotas em Portadores de Diabetes Melito
}

\begin{abstract}
RESUMO
O transplante simultâneo de pâncreas/rim tem indicações específicas, riscos e benefícios. $O$ procedimento, cada vez mais realizado, traz vantagens se comparado ao paciente em diálise, em relação à qualidade de vida, anos de vida ganhos e evolução das complicações crônicas. Se o paciente tiver a opção de realizar o transplante de rim com doador vivo, que apresenta sobrevida semelhante do enxerto e do paciente aos dez anos, o procedimento deverá ser considerado. $\mathrm{O}$ transplante de pâncreas após rim, quando efetivo, pode melhorar a evolução das complicações cardiovasculares, mas em contrapartida provoca maior mortalidade nos primeiros meses após a cirurgia. $O$ transplante isolado de pâncreas também ocasiona a maior mortalidade pósoperatória, resultado da complexidade do procedimento e da imunossupressão. $O$ transplante de ilhotas tem sua indicação para um seleto grupo de diabéticos com instabilidade glicêmica. (Arq Bras Endocrinol Metab 2008; 52/2:355-366)
\end{abstract}

Descritores: Diabetes melito; Pâncreas; Ilhotas; Transplante

\begin{abstract}
Pancreas and Islet Transplantation in Patients with Diabetes Mellitus.

Pancreas and kidney transplants have specific indications, benefits and risks. The procedure has become more common and more often as long-term success has improved and risks have decreased. Compared with a patient being on dialysis, simultaneous pancreas-kidney transplant offers a distinct advantage when it comes to mortality, quality of life and diabetic complications. Since there can be a living-donor kidney transplant, , a possibly similar patient and graft survival by 10 years follow-up, this procedure should be considered. Pancreas after kidney transplants, when successful, can improve microvascular complications compared with kidney transplant alone, but immediate mortality may be higher. Solitary pancreas transplantation can improve the quality of life in selected patients, but it may also increase the immediate risk of mortality due to the complexity of the surgery and the risks of immunosupression. The results of Islet transplantation differ from the higher metabolic performance achieved by whole pancreas allotransplantation and its applicability is limited to selected adult diabetic patients. (Arq Bras Endocrinol Metab 2008;52/2:355-366)
\end{abstract}

Keywords: Diabetes mellitus; Pancreas; Islet; Transplantation

\section{INTRODUÇÃO}

A NTES DE 1922 UM PACIENTE diagnosticado portador de diabetes melito tipo 1 (DMl) tinha uma expectativa de vida ao redor de dois anos. En- revisão

\author{
JoÃo Roberto de Sá \\ ADRIANO MIZIARA GONZALEZ \\ Cláudio Santiago Melaragno \\ DAVID SAITOVICH \\ DENISE REIS Franco \\ ERIKA BEVILAQUA RANGEL \\ IRENE LOURDES NORONHA \\ José Osmar Medina Pestana \\ Marcelo Casaccia Bertoluci \\ MARCELO LINHARES \\ Marcelo Perosa de Miranda \\ Patricia Monteagudo \\ TÉrCIO GENZINI \\ FredDY GoldBerg EliascheWItZ
}

Departamento de Medicina das Disciplinas de Endocrinologia (JRS, PM) e de Nefrologia (CSM, EBR, JOMP) e Departamento de Cirurgia da Disciplina de Gastroenterologia

Cirúrgica (AMG, ML) todos da Universidade Federal de São Paulo (Unifesp), SP; Serviço de Nefrologia do Hospital São Lucas da Pontifícia Universidade Católica do Rio Grande do Sul (PUC-RGS), Porto Alegre, RS (DS); Núcleo de Terapia Celular e Molecular (Nucel) da Universidade de São Paulo (USP) (DRF, FGE, ILN); Centro de Pesquisa Médica Notre-Dame Intermédica Sistema de Saúde, SP (DRF, FGE); Departamento de Clínica Médica da Faculdade de Medicina da USP, SP (ILN); Departamento de Medicina Interna da Universidade Federal do Rio Grande do Sul (UFRGS), Porto Alegre, RS (MCB); Serviço de Transplante de Pâncreas do Hospital Israelita Albert Einstein (HIAE-SP), SP (EBR, MPM, TG) Brasil.

Recebido em 30/01/2008

Aceito em 08/01/2008 
tretanto, o isolamento e a produção em escala industrial da insulina provocaram uma revolução no tratamento da doença, pois a transformou de doença rapidamente fatal em crônica. O aumento da expectativa de vida acarretou o surgimento das complicações crônicas, que ocorrem cerca de 10 a 20 anos após o diagnóstico.

Cerca de um terço dos pacientes com DM desenvolvem doença renal terminal (DRT) (1) e, até metade do século passado, os doentes neste estado tinham poucas alternativas, já que não eram considerados bons candidatos ao transplante de rim pelos cirurgiões por causa do descontrole glicêmico que propiciava, entre outras complicações, o ressurgimento da nefropatia no enxerto renal e por outro lado eram vistos pelos clínicos como de difícil controle metabólico, em virtude da perda progressiva da função renal, ou seja, pouco se fazia para este subgrupo de pacientes. Em 1966, Kelly e cols. (2) realizaram o primeiro transplante de pâncreas em humanos, em uma paciente diabética, que apresentou fístula pancreática como complicação, e enxertectomia do pâncreas e do rim, cerca de dois meses após, com morte por embolia pulmonar. Apesar dos resultados ruins no início do programa, ficou demonstrado que o controle glicêmico sem insulina exógena era possível com o transplante de pâncreas. Com a melhora das técnicas cirúrgicas, da imunossupressão e dos cuidados multidisciplinares envolvidos no transplante de órgãos, os resultados melhoraram progressivamente e, segundo dados atuais, mais de 23 mil transplantes de pâncreas já foram realizados no mundo (3). O Brasil ocupa atualmente lugar de destaque, visto que desde o primeiro transplante realizado no Rio de Janeiro, mais de 800 foram posteriormente feitos, com resultados comparáveis à maioria dos centros internacionais. A modalidade mais realizada em nosso país é o transplante simultâneo de pâncreas e rim $(\mathrm{n}=$ 633), seguido do pâncreas isolado, que inclui tanto o transplante de pâncreas após rim (TPAR) quanto o isolado de pâncreas (TIP) $(\mathrm{n}=217)$, conforme registro da Associação Brasileira de Transplantes de Órgãos (ABTO), no período de janeiro de 1995 a dezembro de 2004.

Os benefícios do transplante do pâncreas são a melhora da qualidade de vida, a prevenção da recorrência da nefropatia diabética, a parada de uso da insulina exógena e da monitoração glicêmica, a liberdade dietética e o potencial benefício em relação às complicações crônicas da doença.

\section{INDICAÇÕES PARA O TRANSPLANTE DE PÂNCREAS}

O paciente com $\mathrm{DMl}$ poderá se beneficiar de três tipos de transplante de pâncreas, que dependerá de sua função renal, da possibilidade de obter um doador de rim vivo relacionado e de sua instabilidade glicêmica. As indicações para o transplante de ilhotas serão discutidas separadamente.

Pacientes com DMl ou insulino-dependentes com doença renal terminal (DRT) podem ser submetidos a: transplante de rim isolado (TRI), transplante simultâneo de pâncreas/rim (TSPR) e transplante de pâncreas após rim (TPAR). Enquanto o enxerto pancreático é obtido quase invariavelmente de doador falecido, o enxerto renal pode advir de doador vivo ou falecido.

$\mathrm{Na}$ maioria dos centros de transplante, o candidato usual para TSPR é um paciente com DMl e DRT ou em fase pré-dialítica, com clearance de creatinina menor ou igual a $20 \mathrm{ml} /$ minuto $/ 1,73 \mathrm{~m}^{2}$, com reserva cardíaca adequada, que não tem opção de doador renal vivo ou prefere receber ambos os órgãos simultaneamente de doador falecido (4).

Menos comumente, o enxerto de pâncreas de doador falecido pode ser transplantado simultaneamente com rim de doador vivo, para se evitar duas hospitalizações, enquanto o paciente se beneficia do transplante renal de doador vivo (5).

Segundo dados da United Network for Organ Sharing (UNOS), o TSPR tem taxas de sobrevida de paciente e de enxerto renal de um ano, semelhantes ou até melhores do que o transplante de rim isolado (TRI) em pacientes com diabetes (6). $\mathrm{O}$ transplante renal isolado de doador vivo tem melhor sobrevida de paciente e de enxerto do que o transplante renal de doador falecido, tanto em diabéticos como para não-diabéticos (7). O transplante simultâneo pâncreas/rim, com doador renal vivo oferece sobrevida semelhante ao transplante de rim isolado com doador renal vivo, com a vantagem do controle glicêmico além do potencial da estabilização ou até da regressão de algumas complicações crônicas do DM.

$O$ candidato usual para transplante de pâncreas após rim (TPAR) é o paciente com DMl com transplante renal prévio, com função estável, boa reserva 
cardíaca, com mau controle glicêmico crônico, apesar de terapia insulínica intensiva, e que deseja se beneficiar da normoglicemia.

O transplante isolado de pâncreas (TIP) é a modalidade menos realizada e suas indicações estão entre as mais discutíveis e menos uniformes entre os centros transplantadores. A American Diabetes Association (ADA) (8), bem como a Sociedade Brasileira de Diabetes $(\mathrm{SBD})$, recomendam o TIP apenas para pacientes com DMl, com boa reserva cardíaca e boa função renal (clearance de creatinina de pelo menos $70 \mathrm{ml} / \mathrm{min}$ ) e sem contra-indicações, que apresentem história de labilidade glicêmica com complicações metabólicas agudas graves, como episódios de cetoacidose de repetição, hipoglicemias graves confirmadas, assintomáticas ou não, apesar de diversas tentativas de manejo por endocrinologista, especialmente se associadas a situações de risco, como as relacionadas a acidentes de trabalho, de trânsito ou risco envolvendo cuidado de crianças.

A existência de problemas clínicos e emocionais graves e comprovadamente incapacitantes associados ao automanejo com insulinoterapia exógena são também indicações do transplante isolado de pâncreas (Tabela l).

\section{Drenagem exócrina pancreática: entérica ou vesical?}

Historicamente, a fístula anastomótica nos pacientes submetidos ao TSPR era associada à rejeição duodenal e foi substituída pela drenagem vesical em 1983 (9). Entretanto, os pacientes passaram a apresentar a alta taxa de infecção do trato urinário, ao redor de $62 \%$. A descoberta de novos imunossupressores, como tacroli- mo e micofenolato mofetil, com conseqüente redução da taxa de rejeição, propiciou novamente a utilização preferencial da drenagem entérica pela maioria dos centros transplantadores, notadamente para o transplante simultâneo de pâncreas/rim (10).

A drenagem entérica é mais fisiológica se comparada à vesical. A primeira está associada à menor ocorrência de infecção de trato urinário e episódios de desidratação, pancreatite por refluxo e acidose metabólica. O principal problema é o risco da fístula entérica e a dificuldade de acesso para biópsia do pâncreas, no caso de drenagem venosa portal.

A principal vantagem da drenagem vesical é a possibilidade de utilizar a amilasúria como parâmetro de rejeição pancreática. Outra vantagem é o acesso a uma biópsia por cistoscopia. As desvantagens são as maiores chances de acidose, infecções do trato urinário, pancreatite de refluxo e cistite hemorrágica (11-13).

No Brasil, os grupos transplantadores optam preferencialmente pela drenagem entérica para os pacientes submetidos a transplante duplo de pâncreas e rim. Os grupos dividem-se na preferência em relação aos que se submetem ao pâncreas após rim e majoritariamente optam pela drenagem vesical nos casos de pâncreas isolado.

\section{Drenagem sistêmica ou portal?}

A drenagem sistêmica produz um estado de hiperinsulinemia periférica, com hipoinsulinemia portal, já que ocorre a perda do efeito da primeira passagem da degradação hepática da insulina $(14,15)$. Alguns autores referem que esta situação poderia ser um agravante para a progressão da doença aterosclerótica.

Tabela 1. Critérios para seleção de candidatos para realização de transplante de pâncreas e pâncreas/rim.

\begin{tabular}{|c|c|}
\hline Indicaçōes & Contra-indicações \\
\hline $\begin{array}{l}\text { - Idade de } 18 \text { a } 60 \text { anos. } \\
\text { - DM1 ou insulino-dependente. } \\
\text { - Doença renal crônica terminal secundária ao DM. } \\
\text { - DM instável apesar de tratamento insulínico intensivo } \\
\text { para o TIP. } \\
\text { - Capacidade para aderir ao tratamento. } \\
\text { - Capacidade para entender os riscos e os benefícios } \\
\text { do procedimento. } \\
\text { - Ausência de contra-indicações. }\end{array}$ & $\begin{array}{l}\text { - Insuficiência cardíaca congestiva (FE < 40\%). } \\
\text { - Infarto agudo do miocárdio recente (<6 meses). } \\
\text { - Doença arterial coronariana não passível de correção. } \\
\text { - Doença arterial periférica extensa. } \\
\text { - Ausência de complicações crônicas. } \\
\text { - Abuso de drogas ilícitas/lícitas. } \\
\text { - Doenças psiquiátricas ou retardo mental que impeçam a } \\
\text { - } \text { - Neompreensão do procedimento. } \\
\text { - Obesidade. }\end{array}$ \\
\hline
\end{tabular}


O controle glicêmico é semelhante em relação as duas técnicas de drenagem $(16,17)$, visto que na drenagem portal o metabolismo lipoprotéico é melhor. Entretanto, a ocorrência de trombose é maior e o acesso para uma biópsia, em caso de necessidade, é mais dificultado (18). Além disso, a drenagem exócrina pancreática necessariamente será entérica.

A maioria dos centros transplantadores opta pela drenagem sistêmica, apesar da venosa portal ser mais fisiológica. Os dados atuais não apontam vantagem de sobrevida do enxerto pancreático para quaisquer das técnicas. Em relação aos benefícios relacionados ao metabolismo lipídico e à ausência da hiperinsulinemia periférica são necessários mais estudos para a sua comprovação.

\section{Esquema de imunossupressão}

Apesar da evolução dessa classe de drogas, o ideal seria o encontro do ponto de equilíbrio entre a imunossupressão eficaz com baixas taxas de rejeição e os mínimos efeitos colaterais deletérios metabólicos, infecciosos e neoplásicos.

Um dos pontos de discussão entre os grupos transplantadores refere-se ao uso ou não da terapia de indução para os pacientes que serão submetidos ao transplante duplo de pâncreas e rim, já que para o isolado e após rim a terapia de indução é realizada. A análise dos dados da literatura (19) mostrou que a indução trouxe vantagens após três anos em relação à sobrevida renal e que deveria, portanto, ser indicada também para este subgrupo de pacientes.

Em relação aos inibidores de calcineurina, os estudos mostram que tanto a ciclosporina quanto o tacrolimo são efetivos, porém outros estudos apontam para uma possível vantagem do tacrolimo com menor taxa de rejeição renal (20) e maior taxa de sobrevida do enxerto pancreático $(21)$.

Em relação aos agentes antiproliferativos, os dados são bastante favoráveis à utilização do micofenolato de mofetil em substituição à azatioprina, por causa da menor ocorrência de rejeição e da maior sobrevida do enxerto $(20,22)$.

\section{TRANSPLANTE DE PÂNCREAS E SOBREVIDA DO PACIENTE}

O parâmetro mais importante de um procedimento invasivo, como o transplante, é seu impacto na sobrevida do paciente. As análises realizadas pelos grandes centros transplantadores ou pelos bancos de registros, como a UNOS e o International Pancreas Transplant Registry (IPTR), mostram uma melhora progressiva tanto na sobrevida do enxerto quanto na do paciente, nas três modalidades de transplante de pâncreas. $\mathrm{Na}$ análise geral dos procedimentos realizados entre 1998 e 1999 , a sobrevida do enxerto após cinco anos do TSPR foi de $69 \%$. Para o TPAR e TIP foi de $58 \%$, com viés de alta. As taxas de sobrevida do enxerto aos dez anos para as cirurgias realizadas entre 1992 e 1993 foram de 46\% para TSPR e $17 \%$ para o TPAR e TIP (3).

Em relação a sobrevida do paciente, para 4.718 pacientes submetidos ao TSPR entre 1988 e 1997 foi encontrada sobrevida de $67 \%$, visto que as principais causas de morte foram doenças cardiovascular com 33,4\%, 7,1\% de cerebrovascular, $21,5 \%$ infecciosa e 3,3\% malignidade (23).

As sobrevidas do enxerto pancreático no transplante de pâncreas após rim em um ano, três anos e cinco anos são $78 \%, 66 \%$ e $57 \%$, respectivamente, enquanto as do paciente são $96 \%$, 90\% e $84 \%$, respectivamente (24). No transplante de pâncreas solitário, as sobrevidas do enxerto pancreático em um, três e cinco anos são $77 \%, 63 \% \mathrm{e}$ $56 \%$, respectivamente, enquanto as do paciente são $96 \%$, $94 \%$ e $91 \%$, respectivamente $(24)$.

Dados da UNOS e do IPTR, de 2.427 TPAR e 1.008 TIP realizados entre 1987 e 2004 mostraram sobrevida do paciente dez anos após de $40 \%$ para o TPAR e 74\% para o TIP para procedimentos realizados entre 1992 e 1993 (3). A causa mais freqüente de morte após 12 meses foi doença cardiovascular nos dois grupos (3). Recentemente, Gruessner e cols., em casuística de 518 transplantes isolados de pâncreas, mostrou sobrevida do paciente aos cinco anos de $90 \%$, com sobrevida do enxerto no primeiro ano acima de $95 \%$, em pacientes com diabetes melito instável (25). Estes dados parecem ser, entre outros, o resultado de avanços nas técnicas cirúrgicas e nos imunossupressores. Em relação a anos de vida ganhos em pacientes submetidos ao transplante isolado de pâncreas e pâncreas após rim, os estudos não mostraram vantagens em relação aos pacientes que permaneceram na lista de espera $(26,27)$.

No Brasil, os dados do Sistema Estadual de Transplantes da Secretaria de Estado de Saúde de São Paulo, referentes ao período de julho de 1997 a dezembro de 2004, são compatíveis com as sobrevidas atuariais do paciente submetido ao TSPR em um e cinco anos de $83 \%$ e $77,3 \%$, respectivamente $(28)$. 


\section{Fatores que influenciam a sobrevida ou a função do enxerto pancreático}

A rejeição aguda pancreática é classificada de acordo com os seguintes critérios (29): grau I - infiltrado linfocítico septal de significado indeterminado; grau 2 grau mínimo: infiltrado linfocítico perivenular associado à endotelite (na ausência do infiltrado, devem estar presentes três dos quatro critérios: infiltrado septal, presença de eosinófilos, até dois focos de infiltrado acinar ou inflamação ductal); grau III (rejeição leve): mais de três focos de infiltrado acinar; grau IV (rejeição moderada): presença de arterite e das características anteriores; grau $\mathrm{V}$ - infiltrado inflamatório linfocítico acinar com necrose celular acinar focal ou confluente.

A suspeição clínica de rejeição aguda baseia-se no aumento das enzimas pancreáticas, especialmente a lipase, não sendo a hiperglicemia um critério diagnóstico (29). No caso da derivação vesical, o aumento maior ou igual a duas vezes da amilase e da lípase sanguínea ou o decréscimo da amilasúria em $40 \%$ a $50 \%$ tem especificidade de $80 \%$ para o diagnóstico de rejeição aguda (30).

A resposta ao tratamento da rejeição aguda, de acordo com a classificação da rejeição, é de $40 \%, 88 \%$, $78 \%, 50 \%$ e $17 \%$ nos graus I a V, respectivamente, sendo a resposta mais efetiva nos graus II e III (29). A resposta à corticoterapia (metilprednisolona $500 \mathrm{mg} \mathrm{a}$ $1.000 \mathrm{mg} / \mathrm{dia}$, por 3 a 5 dias) varia de $36 \%, 86 \%, 68 \%$ e $0 \%$ para os graus I, II, III e IV, respectivamente (29). A resposta ao tratamento com anticorpo policlonal varia de $50 \%, 89 \%, 85 \%, 71 \%$ e $17 \%$ para os graus I, II, III, IV e V, respectivamente (29). Na rejeição grau V, a hiperglicemia persiste apesar do tratamento para a rejeição (29).

A rejeição hiperaguda, atualmente, não é freqüente em razão da realização da prova cruzada ou crossmatch para a detecção de anticorpos anti-HLA pré-formados, enquanto a rejeição humoral vem ganhando importância ao longo do tempo, principalmente após a detecção do fragmento do complemento C4d no endotélio vascular do enxerto pancreático (31).

A sobrevida do enxerto pancreático após o TSPR é influenciada por fatores relacionados ao tempo de transplante, de modo que a perda do enxerto pancreático nas primeiras 24 horas até a primeira semana é secundária à trombose de causa técnica e à rejeição aguda, enquanto as perdas após a primeira semana até o primeiro mês são secundárias àquelas duas causas e também à infecção (32). Após o primeiro mês, as perdas por rejeição aguda e crônica predominam, porquanto após o sexto mês a principal causa de perda do enxerto pancreático é a rejeição crônica, que é classificada também pela análise semiquantitativa da fibrose do parênquima do enxerto pancreático e tem correlação positiva com a sobrevida dele (32).

De acordo com os dados da UNOS, no primeiro ano, $45 \%$ dos pacientes submetidos ao TSPR não apresentam rejeição aguda, 36\% têm rejeição aguda apenas renal, 3\% têm rejeição aguda apenas pancreática e $16 \%$ apresentam rejeição aguda nos dois enxertos (33). Os dados de centros isolados também relatam taxas de rejeição aguda renal após o TSPR de 22,8 a $30 \%$, quando não é utilizada globulina antitimócito $(34,35)$.

A função retardada do enxerto pancreático ocorre em cerca de $30 \%$ dos casos e está associada à idade do doador, acima de 45 anos (36). Mas, ao contrário do que ocorre no transplante renal, parece não ter impacto na sobrevida do enxerto pancreático. Contudo, a própria definição de função retardada do enxerto pancreático é conflitante na literatura, sendo a mais aceita aquela definida como alta hospitalar em uso de insulina.

As principais causas de insucesso do transplante pancreático incluem as perdas técnicas e a rejeição crônica, que ocorrem em cerca de $13 \%$ e $8,8 \%$ dos casos, respectivamente $(37,38)$. As perdas técnicas incluem trombose $(52 \%)$, pancreatite $(20,3 \%)$, infecções $(18,7 \%)$, fístulas $(6,5 \%)$ e sangramento $(2,4 \%)$ e estão associadas ao índice de massa corpórea do receptor maior que $30 \mathrm{~kg} / \mathrm{m}^{2}$, ao tempo de isquemia fria do pâncreas maior que 24 horas e à causa de morte cerebral do doador que não seja atribuída a trauma (38). O TSPR está associado a elevadas taxas de complicações pós-operatórias, de modo que cerca $16 \%$ a $43 \%$ dos pacientes necessitam de relaparotomias para abordagem de complicações do pâncreas (39-41). Além disso, as complicações sépticas são mais freqüentes nos casos de TSPR do que nas outras modalidades de transplante pancreático (40). As principais complicações cirúrgicas estão descritas na Tabela 2 (42).

$\mathrm{Na}$ derivação vesical, a amilasúria é utilizada para monitorização da rejeição aguda. Além disso, é necessária a reposição de bicarbonato, via oral, por causa da perda do bicarbonato com a urina. Em cerca de $10 \%$ a $25 \%$ dos casos de drenagem vesical, é necessária a conversão para a drenagem entérica, seja por fístula vesical $(42 \%)$, uretrite $(23 \%)$, hematúria (19\%), infecção urinária recorrente (11\%), pancreatite de refluxo (3\%) e acidose metabólica de difícil manejo clínico (2\%) (10). Apesar de a derivação vesical estar associada a maiores taxas de com- 
Tabela 2. Complicações cirúrgicas após o transplante de pâncreas com derivação entérica ou vesical.

\begin{tabular}{|c|c|c|c|}
\hline Complicação & Quadro clínico & Diagnóstico & Tratamento \\
\hline Abscesso & $\begin{array}{l}\text { Febre, eritema na ferida operatória, } \\
\text { drenagem de secreção purulenta pela } \\
\text { ferida operatória. Manifesta-se em torno } \\
\text { de 7-14 dias após o transplante }\end{array}$ & $\begin{array}{l}\text { Leucocitose, coleção intra- } \\
\text { abdominal detectada pelo } \\
\text { ultra-som ou tomografia } \\
\text { abdominal }\end{array}$ & $\begin{array}{l}\text { Drenagem percutânea } \\
\text { ou aberta, } \\
\text { antibioticoterapia }\end{array}$ \\
\hline Pancreatite & $\begin{array}{l}\text { Dor e aumento do volume do enxerto } \\
\text { pancreático }\end{array}$ & $\begin{array}{l}\text { Aumento das enzimas } \\
\text { pancreáticas } \\
\text { (amilase e lipase) }\end{array}$ & $\begin{array}{l}\text { Sondagem vesical na } \\
\text { derivação vesical e } \\
\text { ocreotide na derivação } \\
\text { entérica }\end{array}$ \\
\hline Trombose & $\begin{array}{l}\text { Dor, hiperglicemia mudança do } \\
\text { aspecto do líquido do dreno na } \\
\text { derivação entérica e hematúria } \\
\text { na derivação vesical }\end{array}$ & $\begin{array}{l}\text { Ultra-som, Doppler ou } \\
\text { angiorressonância }\end{array}$ & $\begin{array}{l}\text { Enxertectomia. Se } \\
\text { trombose parcial, } \\
\text { considerar trombolítico e } \\
\text { anticoagulação }\end{array}$ \\
\hline $\begin{array}{l}\text { Fístula entérica/ } \\
\text { vesical }\end{array}$ & Dor, peritonite, febre, sepse & $\begin{array}{l}\text { Leucocitose, coleção intra- } \\
\text { abdominal detectada pelo } \\
\text { ultra-som ou tomografia } \\
\text { abdominal }\end{array}$ & $\begin{array}{l}\text { Rafia, drenagem cirúrgica, } \\
\text { antibioticoterapia. Risco } \\
\text { elevado de } \\
\text { enxertectomia }\end{array}$ \\
\hline
\end{tabular}

Adaptado de Zanane e cols. (42).

plicações que a entérica $(62 \%$ a $63 \%$ versus $12 \%$ a $20 \%)$, o nível de evidência é $2 b$ (19). Não há, contudo, diferença na sobrevida do enxerto entre as duas derivações (19). A derivação portal, por sua vez, é ainda assunto de estudo em relação a suas vantagens (19).

Os principais fatores envolvidos na rejeição crônica incluem, além da rejeição aguda, a infecção por citomegalovírus, o retransplante, a modalidade de transplante pancreático (solitário versus simultâneo pâncreas/rim) e uma ou duas incompatibilidades no loci B do Human Leucocyte Antigens (HLA) (37).

Além das perdas técnicas e imunológicas, a sobrevida do enxerto pancreático é influenciada por vários outros fatores clínicos. Entre esses fatores, são incluídos a recorrência auto-imune do DMl (43) e o desenvolvimento de hiperglicemia de causa indeterminada, que está associado à idade acima de 40 anos, ao uso dos imunossupressores, à história familiar de diabetes, à obesidade, à infecção pelo vírus da hepatite $\mathrm{C}$, à cor negra e ao desenvolvimento de SM (44-46). A evolução para a perda da função endócrina após o TSPR e, portanto de diabetes, segue a definição dos critérios da ADA e da World Health Organization (WHO) e está associada à menor sobrevida do paciente e do enxerto, além do aumento do risco cardiovascular $(44,45)$.

Outros fatores que certamente influenciam o aparecimento de hiperglicemia após o TSPR seriam os efei- tos dos imunossupressores e a resistência insulínica secundária aos imunossupressores ou a outras causas, como ganho de peso, história familiar de DM, raça, sexo e idade, além da própria glicotoxicidade.

No transplante renal isolado, o tacrolimo está associado à diminuição da secreção da insulina, o que tem correlação inversa com seu nível sérico (46-48). Nas primeiras três semanas após o transplante, ocorre redução em torno de $50 \%$ na secreção de insulina e do peptídeo C pelo pâncreas nativo $(48,49)$. No pâncreas transplantado, o tacrolimo também reduz a secreção de insulina de maneira dose-dependente, além de promover o edema citoplasmático, a vacuolização e a apoptose das ilhotas (50).

Os corticosteróides, por sua vez, estão associados ao aumento da resistência insulínica $(5 \mathrm{l})$ e à exacerbação da lesão das ilhotas desencadeada pelos inibidores de calcineurina no pâncreas transplantado $(50)$.

Conforme Robertson e cols. (52), o funcionamento das células-beta após o transplante de pâncreas tem as seguintes características: a) relação normal entre a secreção rápida da insulina e a glicemia de jejum, apesar da drenagem venosa sistêmica e da hiperinsulinemia; b) a secreção rápida de insulina estimulada pela glicose $\mathrm{e}$ pela arginina diminui paralelamente ao aumento da glicemia de jejum, embora a resposta à arginina esteja presente quando a resposta à glicose já desapareceu; c) a 
secreção rápida de insulina estimulada pela glicose e pela arginina são fortes preditores da massa de célulasbeta do pâncreas e da reserva de secreção de insulina. Assim, a preservação da secreção rápida de insulina estimulada pela glicose e pela arginina está associada à maior sobrevida do enxerto pancreático por uma a duas décadas, de modo que após este período os pacientes diabéticos submetidos ao transplante de pâncreas solitário, ao transplante de pâncreas após rim e TSPR podem apresentar função endócrina preservada com valores normais da glicemia de jejum, da hemoglobina glicada e do teste de tolerância oral à glicose, o que é refletido por uma boa reserva de massa de células-beta $(52)$.

\section{Complicações crônicas após o transplante de pâncreas}

\section{Nefropatia diabética}

$\mathrm{O}$ risco de complicações microvasculares do diabetes está associado ao controle da glicemia (53) e um dos racionais para a realização do transplante isolado de pâncreas é a proteção renal advinda da euglicemia. $\mathrm{O}$ trabalho clássico de Fioretto e cols. demonstrou que após cinco e dez anos ocorreu melhora histológica da nefropatia diabética nos pacientes submetidos a transplante isolado de pâncreas, que, entretanto, apresentaram, concomitantemente, piora da função renal, provavelmente associada a drogas imunossupressoras (54). Em outro trabalho, Farney e cols. (55) seguiu 97 pacientes submetidos ao TIP e notou que aqueles com clearance creatinina inferior a $55 \mathrm{ml} /$ minuto evoluíram para doença renal terminal, mostrando a importância da seleção rigorosa dos candidatos a este procedimento. Assim, as evidências médicas atuais não suportam a indicação de um TIP para a prevenção da nefropatia diabética, já que a nefrotoxicidade dos imunossupressores, talvez associados à contração de volume em virtude da drenagem vesical, bem como da acidose metabólica, podem a longo prazo comprometer a função renal, que parece ser mais rápida quanto pior é a função renal pré-transplante (56).

\section{Retinopatia diabética}

A maioria dos candidatos a transplante de pâncreas já realizou fotocoagulação com laser em razão da retinopatia, e estas lesões são irreversíveis. A RD pode até piorar em alguns pacientes, por curto período, em geral associada ao controle glicêmico rígido $(\mathbf{5 7}, 58)$. O TSPR está associado à estabilização da $\mathrm{RD}$ em 73,3\% dos pacientes, em compa- ração com $54 \%$ nos casos em que houve perda do enxerto pancreático (59), além da melhora na microcirculação conjuntival em comparação aos pacientes diabéticos nãotransplantados e os pacientes diabéticos transplantados renais $(60)$. No entanto, outros autores não relataram diferença na estabilização da retinopatia entre os pacientes com enxerto pancreático funcionante ou não, especialmente quando a retinopatia encontra-se avançada na ocasião do transplante (61). Nos dados da Universidade Federal de São Paulo (Unifesp), a piora da retinopatia no primeiro ano do transplante esteve associada ao valor do clearance da creatinina, não sendo identificados fatores de risco após o primeiro ano. Os dados sugerem que há uma necessidade cada vez menor de intervenções, ou seja, tendência à estabilização da retinopatia na maioria dos pacientes (dados não publicados).

\section{Doença vascular}

A doença cardiovascular (DCV) é a principal causa de morte em pacientes com diabetes melito. Vários estudos mostram que o controle glicêmico reduz o risco de microangiopatia nos $\mathrm{DMl}$, mas em relação à $\mathrm{DCV}$ não existem muitos estudos prospectivos, com número adequado de pacientes.

Em relação ao transplante de pâncreas, trabalhos têm demonstrado redução de fibrinogênio, D-dimeros, homocisteína, triglicérides, fator de von Willebrand em receptores de pâncreas/rim quando comparados a receptores de rim isolado (62). Outros autores mostraram que cinco anos após o transplante, a ocorrência de doenças vasculares não é diferente entre o TSPR e o transplante renal isolado, enquanto em sete a dez anos os pacientes submetidos ao TSPR têm significativamente menor incidência de infarto agudo do miocárdio $(2,4 \%$ a $16 \%$ versus $17,5 \%$ a $50 \%)$, de acidente vascular cerebral (16\% versus $40 \%)$ e de amputações ( $16 \%$ versus $30 \%)$ (62-65).

A mortalidade por causa cardiovascular em sete anos é menor nos pacientes submetidos ao TSPR do que os pacientes que realizaram transplante de rim isolado e do que os pacientes em lista de espera, isto é, $75 \%$ a $77,4 \%, 56 \%$ a $63 \%$ e $35 \%$ a $39,6 \%$, respectivamente $(64,65)$. Outros autores relatam, no entanto, sobrevida em oito anos do paciente de cerca de $70 \%$ tanto após o TSPR quanto o após o transplante renal (66).

\section{Neuropatia diabética}

A neuropatia diabética está presente em mais de $80 \%$ dos DMl com doença renal terminal (67), sendo difí- 
cil a distinção entre a neuropatia urêmica da diabética (68). Navarro e cols. (69), em estudo com 115 DMl submetidos ao TSPR ou TPAR, compararam estes pacientes com transplantados de rim isolado ou com os que perderam a função do enxerto pancreático. Os pacientes com enxerto de pâncreas funcionante mostraram a rápida melhora nos parâmetros eletrofisiológicos de sensibilidade e condução, sem, entretanto atingir a normalização, mesmo após dez anos de seguimento. Allen e cols. (70), no seguimento de $59 \mathrm{DMl}$ com TSPR por oito anos encontraram resultados muito semelhantes aos de Navarro.

A neuropatia autonômica, cuja presença está associada à maior taxa de mortalidade (68), pode apresentar melhora, mas, em geral, ocorre após um tempo mais prolongado, ao redor de cinco anos (69).

\section{TRANSPLANTE DE ILHOTAS}

O racional para o desenvolvimento do transplante de ilhotas é que estas representam apenas $1 \%$ a $2 \%$ da massa celular do pâncreas, sendo todo o restante do órgão representado por tecido não endócrino, cujo transplante é desnecessário para o paciente com DMl.

\section{Indicações para o transplante de ilhotas}

A indicação do transplante de ilhotas deve considerar os efeitos adversos, tanto do procedimento quanto do uso crônico dos imunossupressores, para que uma cuidadosa análise do risco/beneficio seja feita. A indicação do autotransplante para mitigar ou evitar o diabetes em pacientes submetidos à pancreatectomia subtotal tem sido feita há mais de 30 anos com ótimos resultados. Uma única infusão com cerca de 300 mil IEQs é capaz de impedir o diabetes na maioria dos pacientes, que, neste caso, não sofrem os efeitos deletérios, inclusive diabetogênicos, dos imunossupressores e tampouco apresentem reações de autoimunidade contra as ilhotas implantadas. Outra condição de indicação mais fácil é quando o paciente já está sob imunossupressão em esquema semelhante ao do protocolo de Edmonton e apresenta função renal normal (clearance de creatinina $\geq 80 \mathrm{ml} / \mathrm{min}$ ). As indicações mais difíceis são as do transplante de ilhotas para pacientes DMl sem imunossupressão prévia, nos quais a ponderação do benefício reside em eliminar a labilidade do controle glicêmico, evitando o risco das hipoglicemias inadvertidas (71).

\section{O procedimento do transplante de ilhotas}

As ilhotas são implantadas no fígado por meio de uma infusão na veia porta. $\mathrm{O}$ acesso ao sistema venoso portal se faz pela cateterização transcutânea dirigida por ultra-sonografia. As ilhotas acondicionadas em uma bolsa estéril siliconizada estão suspensas em aproximadamente $250 \mathrm{ml}$ de meio de cultura de células modificado (meio de transplante) contendo heparina. A infusão leva de 20 a 40 minutos para ser completada. Durante este período a pressão venosa portal é monitorizada e o procedimento deve ser interrompido se a pressão portal ultrapassar $20 \mathrm{~mm}$ de água ou atingir o dobro do valor basal. A escolha do fígado como local do implante é decorrente da capacidade elástica deste órgão em acomodar o volume da infusão, do fato que a insulina é fisiologicamente secretada no sangue portal e do sucesso clínico alcançado com este sítio de implante (72-74).

Os eventos adversos sérios mais comuns do procedimento são: alteração das provas de função hepática, sangramento intra-abdominal e trombose dos ramos da veia porta. A morbidade associada ao transplante de ilhotas é 20 vezes menor do que a do transplante de pâncreas (75).

\section{Imunossupressão no transplante de ilhotas}

A imunossupressão recomendada pelo protocolo de Edmonton consiste de daclizumabe, sirolimo e tacrolimo, sem o uso de corticóides. O daclizumabe (anticorpo monoclonal anti-CD25) é administrado por via endovenosa na dose de $1 \mathrm{mg} / \mathrm{kg}$ no dia do implante e em mais quatro aplicações a cada duas semanas após cada infusão. O sirolimo é administrado por via oral uma vez ao dia na dose necessária para manter o nível sanguíneo na faixa de 12 a $15 \mathrm{ng} / \mathrm{ml}$ durante os primeiros três meses; posteriormente a dose é ajustada para manter níveis entre 7 a $12 \mathrm{ng} / \mathrm{ml}$. A dose inicial administrada de sirolimo é de $0,2 \mathrm{mg} / \mathrm{kg}$, a dose subseqüente é de $0,1 \mathrm{mg} / \mathrm{kg}$, que será depois ajustada semanal/quinzenalmente até que as concentrações sanguíneas desejadas sejam atingidas. $\mathrm{O}$ tacrolimo é administrado na dose inicial de 1 a $2 \mathrm{mg} /$ dia; depois é ajustada para manter a concentração sanguínea de 3 a 6 $\mathrm{ng} / \mathrm{ml}$. A terapia imunossupressora é mantida por toda a vida e é importante ressaltar que ela tem efeito antiproliferativo e diabetogênico, tanto por seu efeito inibidor da secreção de insulina quanto por induzir resistência à ação deste hormônio (76-79). 


\section{Resultados do transplante de ilhotas}

Decorridos sete anos da publicação de Lakey e cols. (protocolo de Edmonton), o transplante de ilhotas seguindo este protocolo ou suas variantes foi realizado em mais de 500 pacientes de 53 centros, incluindo o Núcleo de Terapia Celular e Molecular (Nucel), permitindo uma reavaliação crítica dos progressos e dos desafios que o transplante de ilhotas pancreáticas humanas ainda deve superar para migrar do campo da pesquisa clínica para o da terapia estabelecida.

A taxa de sucesso, medida pela insulino-independência em um ano, obtida nos três centros com maior experiência na América do Norte (Edmonton, Miami e Minneapolis) com a infusão de ilhotas obtidas de um a quatro pâncreas é de $82 \%$ (nos demais centros varia de $0 \%$ a $63 \%$ ) e em todos se observa perda progressiva da insulino-independência. Três anos após o transplante, $50 \%$ dos pacientes permanecem livres de insulina e após cinco anos, apenas $13 \%$ dos pacientes não necessitam de insulina para controlar a sua glicemia. No entanto, o peptídeo C continua detectável em $80 \%$ dos pacientes e esta secreção residual da insulina traz o benefício da melhora ou do desaparecimento da labilidade, uma grande redução dos eventos hipoglicêmicos e a manutenção de uma Alc dentro das metas recomendadas $(75,80-82)$.

\section{Conclusões}

O transplante de ilhotas representa a fronteira na inovação tecnológica para o tratamento de um grupo específico de pacientes portadores de DMl. É um procedimento terapêutico aprovado no Canadá, em fase de aprovação nos EUA e experimental nos demais países, incluindo o Brasil. Atualmente, este procedimento não deve ser encarado como um modo seguro de atingir a insulino-independência porque, após cinco anos de seguimento, apenas uma pequena parcela dos pacientes se mantém livre de insulina, embora a maioria se beneficie do desaparecimento da hiperlabilidade. Com o desenvolvimento de novas técnicas de preparação das ilhotas e da imunossupressão dos pacientes, será possível atingir a insulino-independência com a infusão de uma massa celular menor e o transplante de ilhotas poderá oferecer a mesma eficácia clínica que o transplante de pâncreas, se não com menor custo com menor morbidade. Assim como o sucesso do transplante de pâncreas criou a motivação e a oportunidade para que o transplante de ilhotas se desenvolvesse, esta nova modalidade criará a oportunidade e a motivação para o desenvolvimento do transplante utilizando células provenientes de fontes alternativas, como as células-tronco diferenciadas em laboratório, e estimulará o desenvolvimento de técnicas de indução da imunotolerância e de imunoisolamento, porque este é o caminho para tornar a terapia de reposição celular aplicável a uma população maior de pacientes $(78,83,84)$.

\section{CONSIDERAÇÕES GERAIS}

O transplante de pâncreas é um procedimento invasivo que atualmente é o único tratamento que consegue restaurar e manter com alta taxa de sucesso e por tempo prolongado a normoglicemia em portadores de DMI. O procedimento eleva a taxa de morbimortalidade nos primeiros meses após a cirurgia, se comparado principalmente ao transplante de rim com doador vivo, mas traz melhor qualidade de vida aos pacientes.

Embora controverso, vários estudos demonstram estabilização ou melhora de algumas das complicações crônicas do diabetes e mesmo ganho em anos de vida para pacientes submetidos ao transplante duplo de pâncreas e rim.

Estudos recentes têm demonstrado resultados conflitantes em relação ao transplante isolado de pâncreas, o que reforça a necessidade de uma seleção criteriosa de pacientes para este procedimento.

O transplante de ilhotas, atualmente, não deve ser encarado como um modo seguro de atingir a insulinoindependência, porque após cinco anos de seguimento apenas uma pequena parcela dos pacientes se mantém livre de insulina, embora a maioria se beneficie do desaparecimento da hiperlabilidade glicêmica.

\section{REFERÊNCIAS}

1. Deckert T. Insulin-dependent diabetes mellitus and its complications In: Groth C, editor. Pancreatic transplantation. Philadelphia: WB Saunders \& Co; 1988.

2. Kelly WD, Lillehei RC, Merkel FK, Idezuki Y, Goetz F. Allotransplantation of the pancreas and duodenum along with the kidney in diabetic nephropathy. Surgery. 1967;61(6):827-37.

3. Gruessner AC, Sutherland DE. Pancreas transplant outcomes for United States (US) and non-US cases as reported to the United Network for Organ Sharing (UNOS) and the International Pancreas Transplant registry (IPTR) as of June 2004. Clin Transplant. 2005;19(4):433-55.

4. Larsen JL. Pancreas transplantation: indications and consequences. Endocr Rev. 2004;25:(6) 919-46.

5. Farney A, Cho E, Schweitzer E, Dunkin B, Philosophe B, Colonna J, et al. 200 simultaneous cadaver pancreas living-donor kidney transplantation: a new approach for the type 1 diabetic uremic patient. Ann Surg. 2000;232:696-703. 
6. Cecka JM. The UNOS Scientific Renal Transplant Registry. Clin Transpl. 2000;1-18.

7. Reddy KS, Stablein D, Taranto S, Stratta RJ, Johnston TD, Waid $\mathrm{TH}$, et al. Long-term survival following simultaneous kidney-pancreas transplantation versus kidney transplantation alone in patients with type 1 diabetes mellitus and renal failure. Am J Kidney Dis. 2003;41:464-70.

8. American Diabetes Association Positional Statements: pancreas and islet transplantation in type 1 diabetes. Diabetes Care. 2006;29:935.

9. Sollinger HW, Kalayoglu M, Hoffman RM, Deierhoi MH, Belzer FO. Quadruple immunosuppressive therapy in whole pancreas transplantation. Transplant Proc. 1987;19 (1 Pt 3):2297-9.

10. Sollinger HW, Odorico JS, D Álessandro AM, Kalayoglu M, Pirsch JD. Experience with 500 simultaneous pancreas-kidney transplants. Ann Surg. 1998;228:284-96.

11. Sindhi R, Stratta RJ, Lowell JA, Sudan D, Cushing KA, Castaldo $P$, et al. Experience with enteric conversion after pancreatic transplantation with bladder drainage. J Am Coll Surg. 1997;184:281-9.

12. Sollinger HW, Messing EM, Eckhoff DE, et al. Urological complications in 210 consecutive simultaneous pancreas-kidney transplants with bladder drainage. Ann Surg. 1993; 218(4):561-68 [discussion: 568-70].

13. Ploeg RJ, Eckhoff DE, D'Allessandro AM, et al. Urological complications and enteric conversion after pancreas transplantation with bladder drainage. Transplant Proc. 1994;26(2):458-9.

14. Diem P, Abid M, Redmon JB, Sutherland DE, Robertson RP. Systemic venous drainage of pancreas allografs as independent cause of hiperinsulinemia in type I diabetic recipients. Diabetes Care. 1990;39:534-40.

15. Gaber AO, Shokouh-Amiri MH, Hathaway DK, Hammontree L, Kitabchi AE, Gaber LW, et al. Results of pancreas transplantation with portal venous and enteric drainage. Ann Surg. 1995;221:613-22; [discussion 622-4].

16. Robertson RP, Abid M, Sutherland DE, Diem P. Glucose homeostasis and insulin secretion in human recipients of pancreas transplantation. Diabetes 1989:38 (Suppl 1):97-8.

17. Cattral MS, Bigam DL, Hemming AW, et al. Portal venous and enteric exocrine drainage versus systemic venous and bladder exocrine drainage of pancreas grafts: clinical outcome of 40 consecutive transplant recipients. Ann Surg. 2000;232:688-95.

18. Lipshutz GS, Wilkinson AH. Pancreas-Kidney and pancreas transplantation for the treatment of diabetes mellitus. Endocrinol Metab Clin North Am. 2007;36:1015-38.

19. Demartines N, Schiesser M, Clavien PA. An evidence-based analysis of simultaneous pancreas-kidney and pancreas transplantation alone. Am J Transplant. 2005;5:2688-97.

20. Stegall MD, Simon M, Wachs ME, Chan L, Nolan C, Kam I. Mycophenolate mofetil decreases rejection in simultaneous pancreas-kidney transplantation when combined with tacrolimus or cyclosporine. Transplantation. 1997;64:1695-700.

21. Bechstein WO, Malaise J, Saudek F, et al. efficacy and safety of tacrolimus compared with cyclosporine microemulsion in primary simultaneous pancreas-kidney transplantation: 1 year results of a large multicenter trial. Transplantation. 2004;77: 1221-8.

22. Merion RM, Henry ML, Melzer JS, Sollinger HW, Sutherland DE, Taylor RJ. Randomized prospective trial of mycophenolatemofetil versus azathioprine for prevention of acute renal allograft rejection after simultaneous kidney-pancreas transplantation. Transplantation. 2000;70:105-11.
23. Ojo AO, Meier-Kriesche HU, Hanson JA, Leichtman A, Magee JC, Cibrik D, et al. The impact of simultaneous pancreas-kidney transplantation on long-term patient survival. Transplantation. 2001;71:82-90.

24. Cohen DJ, St Martin L, Christensen LL, Bloom RD, Sung RS. Kidney and pancreas transplantation in the United States, 1995-2004. Am J Transplant. 2006;6 (5 Pt 2):1153-69.

25. Gruessner RWG, Sutherland DER, Kandaswany R, Gruessner AC. Over 500 solitary pancreas transplants in nonuremic patients with Britlle diabetes mellitus. Transplantation. 2008;85:42-7.

26. Venstron JM, McBride MA, Rother KI, Hirshberg B, Orchard TJ, Harlam DM. Survival after pancreas transplantation in patients with diabetes and preserved kidney function. JAMA. 2003;290:2817-23.

27. Gruessner RW, Sutherland DE, Gruessner AC. Mortality assessment for pancreas transplants. Am J Transplant. 2004;4:2018-26.

28. Ferraz AAB, Sette, MJA. Transplante de fígado: realidade brasileira. ABCD Arq Bras Cir Dig. 2005;18(4):133-7.

29. Papadimitriou JC, Drachenberg CB, Wiland A, Klassen DK, Fink J, Weir MR, Cangro C, et al. Histologic grading of acute allograft rejection in pancreas needle biopsy: correlation to serum enzymes, glycemia, and response to immunosuppressive treatment. Transplantation. 1998;66(12):1741-5.

30. Klassen DK, Hoen-Saric EW, Weir MR, Papadimitriou JC, Drachenberg $C B$, Johnson $L$, et al. Isolated pancreas rejection in combined kidney pancreas transplantation. Transplantation. 1996;27;61(6):974-7.

31. Melcher ML, Olson JL, Baxter-Lowe LA, Stock PG, Posselt AM. Antibody-mediated rejection of a pancreas allograft. Am J Transplant. 2006;6(2):423-8.

32. Drachenberg CB, Papadmitrou JC, Farney A, Wiland A, Blauth $\mathrm{S}$, Fink JC, et al. Pancreas transplantation: the histologic morphology of graft loss and clinical correlations. Transplantation. 2001;71(12):1784-91.

33. Reddy KS, Davies D, Ormond D, Tuteja S, Lucas BA, Johnston TD, et al. Impact of acute rejection episodes on long-term graft survival following simultaneous kidney-pancreas transplantation. Am J Transplant. 2003;3(4):439-44.

34. Sansalone CV, Maione G, Aseni P, Mangoni I, De Roberto A, Soldano $S$, et al. Surgical complications are the main cause of pancreatic allograft loss in pancreas-kidney transplant recipients. Transplant Proc. 2005;37(6):2651-3.

35. Reddy KS, Stratta RJ, Shokouh-Amiri H, Alloway R, Somerville T, Egidi MF, et al. Simultaneous kidney-pancreas transplantation without antilymphocyte induction. Transplantation. 2000;69(1):49-54.

36. Tan M, Kandaswamy R, Sutherland DE, Gruessner RW, Gruessner AC, Humar A. Risk factors and impact of delayed graft function after pancreas transplants. Am J Transplant. 2004;4:758-62.

37. Humar A, Khwaja K, Ramcharan T, Asolati M, Kandaswamy R, Gruessner RW, et al. Chronic rejection: the next major challenge for pancreas transplant recipients. Transplantation. 2003;76(6):918-23.

38. Humar A, Ramcharan T, Kandaswamy R, Gruessner RW, Gruessner AC, Sutherland DE. Technical failure after pancreas transplants: why grafts fail and the risk factors - a multivariate analysis. Transplantation. 2004;78(8):1188-92.

39. Sansalone CV, Maione G, Aseni P, Mangoni I, De Roberto A, Soldano $S$, et al. Surgical complications are the main cause of pancreatic allograft loss in pancreas-kidney transplant recipients Transplant Proc. 2005;37(6):2651-3. 
40. Wullstein C, Woeste G, Taheri AS, Dette K, Bechstein WO. Morbidity following simultaneous pancreas/kidney transplantation. Chirurg. 2003;74(7):652-6.

41. Martins L, Pedroso S, Henriques AC, Dias L, Sarmento AM, Seca R, et al. Simultaneous pancreas-kidney transplantation: five-year results from a single center. Transplant Proc. 2006;38(6):1929-32.

42. Zanan F, Abreo KD, Levine S, Maley W, Zibani GB. Pancreatic transplantation: evaluation and management. $\mathrm{J}$ Intensive Care. 2004;19:127-39.

43. Esmatjes E, Rodriguez-Villar C, Ricart MJ, Casamitjana R, Martorell J, Sabater L, Astudillo E, et al. Recurrence of immunological markers for type 1 (insulin-dependent) diabetes mellitus in immunosuppressed patients after pancreas transplantation. Transplantation. 1998;66(1):128-31.

44. Davidson J, Wilkinson A, Dantal J, Dotta F, Haller H, Hernandez D, et al. New-onset diabetes after transplantation: 2003 International consensus guidelines. Transplantation. 2003;75 (10):SS3-SS24.

45. Cosio FG, Pesavento TE, Kim S, Osei K, Henry M, Ferguson RM. Patient survival after renal transplantation: IV. Impact of post-transplant diabetes. Kidney Int. 2000;62(4):1440-6.

46. Maes BD, Kuypers D, Messiaen T, Evenepoel P, Mathieu C, Coosemans W, et al. Posttransplantation diabetes mellitus in FK-506-treated renal transplant recipients: analysis of incidence and risk factors. Transplantation. 2001;72(10):1655-61.

47. van Duijnhoven EM, Boots JMM, Christiaans MHL, Wolffenbuttel BHR, van Hooff JP. Influence of Tacrolimus on glucose metabolism before and after renal transplantation: a prospective study. J Am Soc Nephrol. 2001;12:583-8.

48. van Hooff JP, Christiaans MH, van Duijnhoven EM. Evaluating mechanisms of post-transplant diabetes mellitus. Nephrol Dial Transplant. 2004;19 (Suppl 6):vi8-vi12.

49. van Duijnhoven EM, Christiaans MH, Boots JM, Nieman FH, Wolffenbuttel BH, van Hooff JP. Glucose metabolism in the first 3 years after renal transplantation in patients receiving tacrolimus versus cyclosporine-based immunosuppression. J Am Soc Nephrol. 2002;13(1):213-20.

50. Drachenberg CB, Klassen DK, Weir MR, Wiland A, Fink JC, Bartlett ST, et al. Islet cell damage associated with tacrolimus and cyclosporine: morphological features in pancreas allograft biopsies and clinical correlation. Transplantation. 1999;68(3):396-402.

51. Midtvedt K, Hjelmesaeth J, Hartmann A, Lund K, Paulsen D, Egeland $\mathrm{T}$, et al. Insulin resistance after renal transplantation: the effect of steroid dose reduction and withdrawal. J Am Soc Nephrol. 2004;15(12):3233-9.

52. Robertson RP, Sutherland DER, Lanz KJ. Normoglycemia and preserved insulin secretory reserve in diabetic patients 10-18 years after pancreas transplantation. Diabetes. 1999;48:1737-40.

53. The Diabetes Control and Complications Trial Research Group. The effect of intensive treatment of diabetes on the development and progression of long-term complications in insulindependent diabetes mellitus. N Engl J Med. 1993;329:977-86.

54. Fioretto P, Steffes MW, Sutherland DE, Goetz FC, Mauer M. Reversal of lesions of diabetic nephropathy after pancreas transplantation. N Engl J Med. 1998;339:69-75.

55. Farney AC, Borboroglu PG, Young C, et al. Native renal function after pancreas transplant alone. J Am Soc Nephrol. 2002;13:188A.
56. Mazur MJ, Rea DJ, Griffin MD, et al. Decline in native renal function early after bladder-drained pancreas transplantation alone. Transplantation. 2004;77:844.

57. Landgraf R, Nusser J, Muller W, landgraf-Leurs MM, Thurau S, Ulbig $\mathrm{M}$, et al. Fate of late complications in type I diabetic patients after successful pancreas-kidney transplantation. Diabetes. 1989;38:(Suppl 1):33-7.

58. Ulbig M, Kampik A, Thurau S, Landgraf R, Land W. Long-term follow-up of diabetic retinopathy for up to 71 months after combined renal and pancreatic transplantation. Graefes Arch Clin Exp Ophthalmol. 1991;229:242-5.

59. Konigsrainer A, Miller K, Steurer W, Kieselbach G, Aichberger $C$, Ofner $D$, et al. Does pancreas transplantation influence the course of diabetic retinopathy? Diabetologia. 1991;34(Suppl 1):S86-S88.

60. Cheung AT, Perez RV, Chen PC. Improvements in diabetic microangiopathy after successful simultaneous pancreas-kidney transplantation: a computer-assisted intravital microscopy study on the conjunctival microcirculation. Transplantation. 1999;68(7):927-32.

61. Scheider A, Meyer-Schwickerath E, Nusser J, Land W, Landgraf R. Diabetic retinopathy and pancreas transplantation: a 3-year follow-up. Diabetologia. 1991;34(Suppl 1):S95-S99.

62. Larsen JL, Lynch T, Al'Halawani M, et al. Carotid intima-media thickness by ultrasound measurement in pancreas transplant candidates. Transplant Proc. 1995;27(6):2996.

63. Biesenbach G, Konigsrainer A, Gross C, Margreiter R. Progression of macrovascular diseases is reduced in type 1 diabetic patients after more than 5 years successful combined pancreas-kidney transplantation in comparison to kidney transplantation alone. Transpl Int. 2005;18(9):1054-60.

64. La Rocca E, Fiorina P, Astorri E, Rossetti C, Lucignani G, Fazio $F$, et al. Patient survival and cardiovascular events after kidneypancreas transplantation: comparison with kidney transplantation alone in uremic IDDM patients. Cell Transplant. 2000;9(6):929-32.

65. La Rocca E, Fiorina P, Di Carlo V, Astorri E, Rossetti C, Lucignani $G$, et al. Cardiovascular outcomes after kidney-pancreas and kidney-alone transplantation. Kidney Int. 2001;60 (5):1964-71.

66. Reddy KS, Stablein D, Taranto S, Stratta RJ, Johnston TD, Waid $\mathrm{TH}$, et al. Long-term survival following simultaneous kidneypancreas transplantation versus kidney transplantation alone in patients with type 1 diabetes mellitus and renal failure. Am J Kidney Dis. 2003;41(2):464-70.

67. Kennedy WR, Navarro X, Sutherland SER. Neuropathy profile of diabetic patients in a pancreas transplantation program. Neurology. 1995;45:773-80.

68. Hopt UT, Drognitz O. pancreas organ transplantation short and lon-term results in terms of diabetes control. Curr Concepts Surg. 2000;385:379.

69. Navarro X, Sutherland DER, Kennedy WR. Long-term effects of pancreatic transplantation on diabetic neuropathy. Ann Neurol. 1997; 42:727-36.

70. Allen RDM, Al-Harbi IS, Morris JGL, et al. Diabetic neuropathy after pancreas transplantation: determinants of recovery. Clin. Transplant. 1997;63:830.

71. Merani S, Shapiro AM. Current status of pancreatic islet transplantation. Clin Sci (Lond). 2006;110:611-25.

72. Eliaschewitz FG, Aita CA, Genzini T, et al. First Brazilian pancreatic islet transplantation in a patient with type 1 diabetes mellitus. Transplant Proc. 2004;36:1117-8. 
73. Shapiro AM, Lakey JR, Rajotte RV, et al. Portal vein thrombosis after transplantation of partially purified pancreatic islets in a combined human liver/islet allograft. Transplantation. 1995;59:1060-3

74. Walsh TJ, Eggleston JC, Cameron JL. Portal hypertension, hepatic infarction, and liver failure complicating pancreatic islet autotransplantation. Surgery. 1982;91:485-7.

75. Collaborative Islet Transplantation Registry, http://spitfire.emmes.com/study/isl/index.html, 2007.

76. Gray DW. Avoiding damage to transplanted human islets during implantation is important. Transplantation. 2005;79:1294-5.

77. Shapiro AM, Lakey JR, Ryan EA, et al. Islet transplantation in seven patients with type 1 diabetes mellitus using a glucocorticoid-free immunosuppressive regimen. N Engl J Med. 2000;343:230-8.

78. Tattersall RB. Brittle diabetes revisited: the Third Arnold Bloom Memorial Lecture. Diabet Med. 1997;14:99-110.

79. Hering BJ, Kandaswamy R, Harmon JV, et al. Transplantation of cultured islets from two-layer preserved pancreases in type 1 diabetes with anti-CD3 antibody. Am J Transplant. 2004:4:390-401.
80. Ryan EA, Paty BW, Senior PA, et al. Five-year follow-up after clinical islet transplantation. Diabetes. 2005;54:2060-9.

81. Froud T, Ricordi C, Baidal DA, et al. Islet transplantation in type 1 diabetes mellitus using cultured islets and steroid-free immunosuppression: Miami experience. Am J Transplant. 2005;5:2037-46.

82. Kempf MC, Andres A, Morel P, et al. Logistics and transplant coordination activity in the GRAGIL Swiss-French multicenter network of islet transplantation. Transplantation. 2005;79:1200-5.

83. Bertuzzi F, Ricordi C. Prediction of clinical outcome in islet allotransplantation. Diabetes Care. 2007;30:410-7.

84. Nath DS, Hering BJ. Islet cells replacement therapy. Clin Lab Med. 2005;25:541-56.

\section{Endereço para correspondência:}

João Roberto de Sá

Rua Botucatu, 740

04023-900 Vila Clementino, São Paulo, SP

E-mail: jrsa@uoll.com.br 